\title{
Eficácia, Segurança e Aderência ao Tratamento de Longo Prazo com Hormônio de Crescimento (GH) em Adultos com Deficiência de GH
}

\begin{abstract}
RESUMO
Objetivo: Avaliar a eficácia, a segurança e a aderência de quatro anos de tratamento com GH em 18 adultos [ 12 mulheres, 6 homens, com idade média de 50,5 anos (25-66 anos)] com deficiência grave de GH (DGH). Métodos: Avaliações clínica, laboratorial e de composição corporal (DXA) realizadas antes e anualmente após o início do $\mathrm{GH}$, e ecocardiografia realizada antes e após quatro anos de tratamento. Dose de $0,2 \mathrm{mg} \mathrm{GH} /$ dia mantida fixa no primeiro ano, com posteriores ajustes para normalizar IGF-1. Resultados: Redução significativa da gordura corporal total (média 2,8 kg) e da gordura truncal (média 1,9 kg), associadas com aumento da massa magra (média 0,8 $\mathrm{kg}$ ) e aumento da densidade mineral óssea (DMO) em coluna lombar e fêmur, particularmente nos sítios com T-escore menor que 1,0 na avaliação basal. Houve piora dos níveis de insulina e HOMA no primeiro ano de terapia, mas ao final do quarto ano os valores de glicose, insulina, HOMA e hemoglobina glicosilada não eram diferentes dos basais. Desenvolveram diabetes tipo 2 no seguimento dois pacientes com intolerância à glicose pré-tratamento. 0 colesterol total e o LDL colesterol reduziram significativamente, e as mudanças foram proporcionais aos valores basais. Os parâmetros ecocardiográficos não se alteraram. Os efeitos colaterais foram leves e bem tolerados. Não foi observada recorrência tumoral. Baixa adesão ao tratamento (estimada por níveis baixos de IGF-1) ocorreu em quatro (22\%), dois $(11 \%)$ e seis $(30 \%)$ pacientes ao final do segundo, terceiro e quarto ano, respectivamente. Conclusões: Quatro anos de tratamento com GH em adultos com DGH teve impacto positivo sobre a composição corporal, a DMO e o perfil lipídico, e nenhum efeito sobre sensibilidade insulínica e o coração. A intolerância à glicose deve ser cuidadosamente monitorada no tratamento de longo prazo. (Arq Bras Endocrinol Metab 2008; 52/5:879-888)
\end{abstract}

Descritores: Deficiência de GH; Hipopituitarismo; Composição corporal; Doenças cardiovasculares

\section{ABSTRACT}

Efficacy, Safety and Compliance of Long-Term Growth Hormone (GH) Replacement Therapy in Adults With GH Deficiency.

Aim: To study efficacy, safety and compliance of GH therapy for 4 years in 18 GH deficient (GHD) adults [12 women; mean age 50.5 yrs (25-66 yrs)]. Methods: Clinical, biochemical and body composition (DXA) measurements were performed before and every year after GH therapy. Ecocardiography was performed at baseline and after 4 years. Dose of $\mathrm{GH}$ was $0.2 \mathrm{mg} /$ day during the first year with subsequent titration to attain normal IGF-1 levels. Results: There was a significant reduction of total body fat (mean $2.8 \mathrm{~kg}$ ), truncal fat (mean $1.9 \mathrm{~kg}$ ) and an increase of lean body mass (mean $0.8 \mathrm{~kg}$ ) and bone mineral density (BMD) on lumbar spine and femur, particularly in sites with T-score $<-1,0$ at baseline. Insulin levels and HOMA index worsened in the first year, but at the end no changes were noted on glucose, insulin, HOMA

\section{artigo original}

\author{
Daniele C. T. Zaninell \\ LUDIMYLA H. F. MEISTER \\ ROSANA B. RADOMINSKI \\ VICTORIA Z. C. BORBA \\ Admar Moraes Souza \\ CESAR L. BOGUSZEWSKI
}

Serviço de Endocrinologia e

Metabologia do Paraná (SEMPR)

do Hospital de Clínicas da

Universidade Federal do Paraná

(UFPR), Curitiba, PR, Brasil (DCTZ,

LHFM, RBR, VZCB, CLB); Disciplina

de Cardiologia do

Departamento de Clínica

Médica do Hospital de Clínicas da UFPR, Curitiba, PR, Brasil (AMS).
Recebido em 4/5/2008

Aceito em 22/5/2008 
index and glycosylated hemoglobin. Two patients with altered glucose tolerance at baseline developed type 2 diabetes during follow-up. Total and LDLcholesterol were significantly lower after therapy, with changes directly associated with baseline values. Cardiac parameters did not change. Side effects were mild and disappeared spontaneously. Tumor recurrence was not observed. Low compliance (estimated by low IGF-1 levels) was observed in 4 $(22 \%), 2(11 \%)$ and $6(33 \%)$ patients at the end of second, third and fourth year, respectively. Conclusions: Four years of GH therapy in GHD adults had a positive impact on body composition, BMD and lipid profile, with no effects on insulin sensitivity and heart. Glucose tolerance should be monitored carefully during long-term GH therapy. (Arq Bras Endocrinol Metab 2008; 52/5:879-888)

Keywords: GH deficiency; Hypopituitarism; Body composition; Cardiovascular disease

\section{INTRODUÇÃO}

$\mathrm{N}$ AS DUAS ÚlTIMAS DÉCADAS, o hormônio de crescimento $(\mathrm{GH})$ recombinante humano tem sido amplamente utilizado no tratamento de reposição da deficiência de GH em adultos (DGHA). Quando esta terapia foi introduzida na prática clínica, vários estudos duplo-cegos e controlados por placebo demonstraram que a reposição de $\mathrm{GH}$ era capaz de promover alterações benéficas na composição corporal, na densidade mineral óssea (DMO), nos fatores de risco cardiovascular e na qualidade de vida, especialmente nos pacientes com DGHA mais intensa do ponto de vista clínico e laboratorial (1-4). Entretanto, os dados sobre a eficácia e a segurança da terapia prolongada com GH na DGHA são mais escassos e podem ser influenciados por inúmeros fatores, como o envelhecimento, o sexo, o nível de hormônios esteróides, o índice de massa corporal (IMC), os determinantes genéticos e o próprio esquema terapêutico empregado. Além disso, os benefícios da terapia de longo prazo devem ser balanceados com os potenciais riscos do uso de GH sobre a tolerância à glicose e à sensibilidade insulínica, os fatores de risco cardiovascular e a recorrência tumoral.

O envelhecimento está associado a ganho de peso, mudanças desfavoráveis na composição corporal e redução da sensibilidade à insulina, e o efeito da idade deve ser considerado nos efeitos da terapia prolongada com GH (5). Estudos prévios sugerem que o tratamento com $\mathrm{GH}$ pode prevenir a piora da sensibilidade à insulina relacionada à idade na $\operatorname{DGHA}(6,7)$. Por outro lado, a suscetibilidade individual e a possibilidade de tratamento com doses excessivas durante terapia prolongada poderiam contribuir para a piora da sensibili- dade à insulina (8-10). Por esses motivos, o efeito global do $\mathrm{GH}$ na sensibilidade à insulina na DGHA ainda é assunto de controvérsias na literatura.

A avaliação dos efeitos do $\mathrm{GH}$ sobre a massa óssea é influenciada por fatores como idade, sexo e período de instalação da DGHA. A DMO é afetada particularmente em pacientes jovens com DGHA de início na infância (11), e há maior risco de fraturas nos pacientes com DGHA do que na população geral (12). O efeito do $\mathrm{GH}$ parece ser bifásico, com maior reabsorção óssea na fase inicial de tratamento seguida de estímulo para formação óssea, com o efeito anabólico prevalecendo no longo prazo (13).

O hipopituitarismo está associado com o aumento da mortalidade cardiovascular, e DGHA sem tratamento é uma das possíveis causas para explicar esta relação (14). A DGHA caracteriza-se pelo aumento nas concentrações séricas de colesterol total, triglicerídeos e lipoproteínas de baixa densidade (LDL), além de redução dos níveis de lipoproteínas de alta densidade (HDL). De modo geral, os estudos de reposição com GH demonstram efeitos favoráveis sobre o colesterol total e o LDL, mas sem repercussões significativas nos triglicerídeos (1-4). Além disso, o volume de ejeção cardíaco e a distensão arterial tendem a ser diminuídos nos pacientes com DGHA, embora os efeitos da terapia com GH sobre estas alterações não estejam totalmente comprovadas (15).

Neste estudo prospectivo, aberto, não controlado, apresentamos nossa experiência clínica de quatro anos de seguimento de um grupo de pacientes com DGHA, que foi tratado com dose baixa fixa de $\mathrm{GH}(0,2 \mathrm{mg} /$ dia) durante o primeiro ano, seguido por ajustes da dose com o objetivo de normalização dos níveis de IGF-1 durante os anos subseqüentes. 


\section{PACIENTES E MÉTODOS}

\section{Pacientes}

Foram estudados 18 pacientes (12 mulheres e seis homens; idade 50,5 $\pm 13,2$ anos, variação entre 22 e 66 anos) com diagnóstico de DGHA, confirmado pelo pico de resposta ao $\mathrm{GH}$ menor que $3 \mu \mathrm{g} / \mathrm{L}$ durante teste de tolerância à insulina (TTI). Desses pacientes, quatro tinham DGHA de instalação na infância (idiopático, craniofaringeoma, deficiência de Pit-1 e sela vazia) e 14 tinham DGHA de instalação na vida adulta (cinco com síndrome de Sheehan, dois com craniofaringieoma, um com prolactinoma, três com adenoma hipofisário não-funcionante, um com trauma, um com sela vazia e um paciente com DGHA após tratamento para acromegalia). Apresentava DGHA isolada um paciente e tinham hipopituitarismo completo 14 pacientes (incluindo três com diabetes insípido), enquanto em outros três pacientes, a DGHA estava associada com deficiência de TSH, TSH + LH/FSH e TSH + ACTH. Os pacientes foram tratados com a terapia de reposição convencional quando indicado. Algumas mulheres em idade de menopausa não estavam recebendo esteróides sexuais em razão de preferências individuais. Tinham feito uso de GH para tratamento de baixa estatura na infância dois pacientes, mas o tratamento já havia sido suspenso há pelo menos dez anos em ambos os casos. Consentimento informado foi obtido de todos os pacientes, e o estudo foi aprovado pelo Comitê de Ética da nossa instituição.

\section{Métodos}

Todos os pacientes foram tratados com injeções subcutâneas diárias de GH recombinante humano (Norditropin e Norditropin Simplexx ${ }^{\circledR}$, Novo Nordisk Farmacêutica, São Paulo, Brasil) aplicado à noite. No primeiro ano, o GH foi aplicado em dose fixa de 0,2 $\mathrm{mg} /$ dia. Nos anos subseqüentes, a dose de GH foi ajustada com o objetivo de atingir níveis normais de IGF-1 para idade e sexo. Os pacientes não foram estimulados a mudar seu padrão de dieta ou atividade física. Ao serem incluídos no estudo, os pacientes foram submetidos a exame físico (incluindo parâmetros antropométricos) e amostras de sangue foram coletadas para exames laboratoriais (perfil bioquímico e hormonal). Todos os participantes foram submetidos à medida da absorção de raio-X de dupla energia (DXA) para avaliação de DMO e composição corporal, além de ecocardiografia. Foram realizadas consultas 30, 90 e
180 dias após o início do tratamento, e a cada seis meses subseqüentemente, e os pacientes, a cada visita, foram sempre questionados sobre a melhora clínica e os efeitos colaterais. Toda a avaliação foi repetida após $12,24,36$ e 48 meses do início do tratamento, exceto ecocardiografia, que foi realizada somente ao final do período do estudo. A ressonância magnética de hipófise foi repetida anualmente nos pacientes com história prévia de tumor.

\section{Dosagens laboratoriais}

As coletas de sangue foram realizadas sempre no período da manhã, após 8 horas de jejum. As dosagens de GH e IGF-1 foram realizadas de acordo com a rotina de atendimento e os métodos variaram conforme o laboratório onde as amostras foram processadas. Assim, $\mathrm{o}$ GH foi dosado pelos métodos de quimioluminescência e imunoradiométrico, enquanto os valores de IGF-1 foram determinados por meio de RIA, quimioluminescência e imunoradiometria. As faixas de referência de normalidade do IGF-1 para sexo e idade foram consideradas de acordo com o método de análise e as informações do fabricante. Para fins de padronização, foi calculado o desvio-padrão (DP) do IGF-1, expresso em $\mathrm{ng} / \mathrm{ml}$, porquanto escores entre $-2 \mathrm{DP}$ e $+2 \mathrm{DP}$ foram considerados normais. Nos pacientes em que os resultados do IGF- 1 estavam expressos em $\mathrm{U} / \mathrm{ml}$, o cálculo do DP não pôde ser realizado. Para cálculo do DP utilizou-se a fórmula (16):

Homens, 18 a 28 anos: IGF-1 DP =

$\frac{\log (\text { IGF- } 1)-3,59686+0,07981 \times \text { idade }-0,001212 \times(\text { idade })^{2}}{0,24928-0,01419 \times \text { idade }+0,0003595 \times(\text { idade })^{2}}$

Mulheres, 18 a 28 anos: IGF-1 DP =

$\frac{\log (\text { IGF- } 1)-3,64235+0,08376 \times \text { idade }-0,001294 \times(\text { idade })^{2}}{0,21944-0,01178 \times \text { idade }+0,0003119 \times(\text { idade })^{2}}$

Homens e mulheres, 29 a 70 anos: IGF-1 DP =

$$
\frac{\log (\text { IGF- } 1)-2,36031+0,002393 \times \text { idade }}{0,137825+0.0001846 \times \text { idade }}
$$

As dosagens de glicemia, insulina, colesterol, HDL e triglicerídeos foram realizadas pelos métodos de rotina em nosso laboratório. Os valores de LDL-colesterol foram calculados por meio da fórmula de Friedewald (17). A resistência à insulina foi estimada clinicamente antes e após o tratamento com GH por intermédio do homeostasis model assessment (HOMA), de acordo com a equação: HOMA-IR = insulina $(\mu \mathrm{U} / \mathrm{ml}) \times$ glicemia $(\mathrm{mmol} / \mathrm{l}) / 22,5(18)$. 


\section{Composição corporal e densidade mineral óssea}

O IMC foi calculado a partir do peso dividido pelo quadrado da estatura $\left(\mathrm{kg} / \mathrm{m}^{2}\right)$. A circunferência abdominal foi aferida no ponto entre a margem inferior da última costela e a crista ilíaca, pelo mesmo investigador, com uma fita métrica inelástica de $0,5 \mathrm{~cm}$ de largura. A medida foi repetida duas vezes, anotando-se a média dos dois valores. A composição corporal (gordura corporal total, gordura truncal e massa magra) e a DMO foram avaliadas por DXA (aparelho Lunar DPX; Madison WI), com coeficiente de variação para medida de composição corporal de $1 \%$. A DMO foi analisada nos seguintes sítios: coluna lombar, colo de fềmur e fềmur total. Para a DMO em coluna lombar foi considerado o valor médio das densidades obtidas nas vértebras $\mathrm{Ll}, \mathrm{L} 2, \mathrm{~L} 3$ e L4. Os resultados da DMO foram expressos em valores absolutos $\left(\mathrm{g} / \mathrm{cm}^{2}\right)$, e em DP em relação à população normal de adulto jovem (T-escore) e uma população pareada por sexo, raça, peso e altura (Z-escore). O coeficiente de variação foi de $1,2 \%$ para a coluna lombar, $1,2 \%$ para o fềmur total e 1,5\% para o colo de fêmur.

\section{Ecocardiografia}

As medidas de ecocardiografia foram realizadas segundo as recomendações da American Society of Echocardiography (19) no início e após 48 meses de tratamento. Os parâmetros avaliados foram: dimensão de raiz aórtica, dimensão de átrio esquerdo ( $\mathrm{AE})$, dimensão diastólica de ventrículo direito (DDVD), espessura diastólica de septo ventricular (EDSV), dimensão diastólica de parede posterior do ventrículo esquerdo (DDPVE), dimensão diastólica final do ventrículo esquerdo (DDVE), dimensão sistólica do ventrículo esquerdo (DSVE), encurta- mento percentual do ventrículo esquerdo, fração de ejeção (FE) do ventrículo esquerdo e massa do ventrículo esquerdo (MVE). $\mathrm{O}$ índice de massa do ventrículo esquerdo (IMVE) foi calculado empregando-se a fórmula da American Society of Ecochardiography (20): IMVE $=\mathrm{MVE} /$ superfície corporal, em que MVE $=0,8(1,04$ [(EDSV + DDVE + DDPVE $) 3-$ DDVE3 $])+0,6$ g.

\section{Análise estatística}

Os resultados são expressos como média $\pm \mathrm{DP}$ ou mediana e valores mínimo e máximo. A diferença entre as variáveis contínuas medidas nos momentos basal, um, dois, três e quatro anos foi avaliada pela aplicação do modelo de análise da variância para medidas repetidas e análise da variância de Friedman, na dependência da presença de normalidade ou não na distribuição da variável estudada. A diferença entre as variáveis contínuas medidas nos momentos basal e aos quatro anos foi avaliada pela aplicação do teste $t$ de Student para amostras pareadas de distribuição normal e teste de Wilcoxon para variáveis de distribuição assimétrica. A associação entre as variáveis contínuas foi estudada por meio do teste de correlação de Pearson. As diferenças foram consideradas estatisticamente significativas para $\mathrm{p}<0,05$.

\section{RESULTADOS}

\section{Dose de GH}

O GH foi administrado em uma dose fixa de $0,2 \mathrm{mg} /$ dia (0,6 UI/dia) durante o primeiro ano de estudo. A dose máxima utilizada foi de $0,66 \mathrm{mg} / \mathrm{dia}$, e a dose média, ao final do segundo, terceiro e quarto anos de tratamento, foi de $0,41,0,45$ e $0,45 \mathrm{mg} /$ dia, respectivamente (Tabela $\mathrm{l}$ ).

Tabela 1. Dose de GH durante tratamento de reposição em 18 pacientes com DGHA e os efeitos do tratamento sobre o peso, IMC, circunferência abdominal (CA), gordura corporal total (GCT), gordura de tronco (GT), massa magra (MM), níveis séricos de IGF-1 e os escores de IGF-1 (IGF-1 DP).

\begin{tabular}{|c|c|c|c|c|c|}
\hline & Basal & $1^{\circ}$ ano & $2^{\circ}$ ano & $3^{\circ}$ ano & $4^{\circ}$ ano \\
\hline Dose GH (mg/dia) & 0,2 & 0,2 & 0,41 & 0,45 & 0,45 \\
\hline Peso (kg) & $72,1 \pm 14,2$ & $71,3 \pm 14,0$ & $70,0 \pm 13,8$ & $70,8 \pm 13,0$ & $70,3 \pm 13,4$ \\
\hline $\operatorname{IMC}\left(\mathrm{kg} / \mathrm{m}^{2}\right)$ & $29,1 \pm 4,8$ & $28,8 \pm 4,7$ & $28,3 \pm 4,9$ & $28,7 \pm 4,9$ & $28,4 \pm 4,4$ \\
\hline $\mathrm{CA}(\mathrm{cm})$ & $95,6 \pm 10,2$ & $94,5 \pm 10,5$ & $94,0 \pm 9,5$ & $94,3 \pm 10,8$ & $93,4 \pm 9,7$ \\
\hline GCT (\%) & $34,8 \pm 10,5$ & $32,6 \pm 10,1^{*}$ & $31,2 \pm 9,8^{*}$ & $32,5 \pm 11,0^{*}$ & $31,8 \pm 10,3^{*}$ \\
\hline GCT (kg) & $24,4 \pm 8,4$ & $22,4 \pm 7,4^{*}$ & $21,3 \pm 6,7^{\circ}$ & $22,4 \pm 7,5^{*}$ & $21,5 \pm 8,0^{*}$ \\
\hline GT (\%) & $36,8 \pm 10,1$ & $34,8 \pm 9,8^{*}$ & $32,6 \pm 8,9^{*}$ & $33,7 \pm 9,9^{*}$ & $33,1 \pm 9,4^{*}$ \\
\hline GT (kg) & $12,3 \pm 4,1$ & $11,8 \pm 3,6$ & $10,6 \pm 3,2^{*}$ & $10,7 \pm 3,2^{*}$ & $10,4 \pm 3,9^{*}$ \\
\hline $\mathrm{MM}(\mathrm{kg})$ & $43,7 \pm 13,0$ & $45,1 \pm 13,4^{*}$ & $46,3 \pm 13,4^{*}$ & $45,6 \pm 13,8^{*}$ & $44,5 \pm 11,6$ \\
\hline IGF-1 (ng/ml) & $64,6 \pm 55,9$ & $111,5 \pm 82,6^{*}$ & $209,3 \pm 160,2^{*}$ & $254,0 \pm 148,8^{*}$ & $178,6 \pm 142,8$ \\
\hline IGF-1 DP & $-4,7 \pm 3,5$ & $-3,2 \pm 3,8^{*}$ & $-0,34 \pm 2,4^{*}$ & $-0,54 \pm 1,9^{*}$ & $-1,1 \pm 3,1^{*}$ \\
\hline
\end{tabular}




\section{Níveis séricos de IGF-1 e IGF-1 DP durante o tratamento com GH}

Os níveis séricos médios pré-tratamento de IGF-1 eram de $64,6 \pm 55,9 \mathrm{ng} / \mathrm{ml}$ e IGF-1 DP de $-4,7 \pm 3,5$. No tempo basal, 11 pacientes $(56 \%)$ apresentavam IGF-1 $\mathrm{DP}<-2$. Houve aumento significativo dos níveis de IGF-1 e IGF-1 DP durante o período do estudo $(\mathrm{p}=$ $0,01)$, com níveis médios ao final do primeiro, segundo, terceiro e quarto anos de tratamento de $111,5 \pm 82,6$ $\mathrm{ng} / \mathrm{ml}(-3,2 \pm 3,8), 209,3 \pm 160,2 \mathrm{ng} / \mathrm{ml}(-0,34 \pm$ $2,4), 254,0 \pm 148,8 \mathrm{ng} / \mathrm{ml}(-0,54 \pm 1,9)$ e $178,6 \pm$ $142,8 \mathrm{ng} / \mathrm{ml}(-1,1 \pm 3,1)$, respectivamente (Tabela 1$)$. A aderência ao tratamento foi avaliada por IGF-1 DP ao final de cada ano de tratamento, exceto o primeiro ano em que foi feito regime de dose fixa. Os escores de IGF-1 $\mathrm{DP}<-2$ foram observados em quatro $(22 \%)$, dois $(11 \%)$ e seis $(33 \%)$ pacientes ao final do segundo, terceiro e quarto anos de tratamento, respectivamente.

\section{Efeitos do tratamento com GH na composição corporal}

Não houve variação significativa no peso corporal, no IMC e na CA com o tratamento. A gordura total reduziu de $34,8 \pm 10,5 \%$ para $31,8 \pm 10,3 \%(\mathrm{p}<0,01)$, equivalente à perda média de $2,9 \mathrm{~kg}$ de gordura total com o tratamento (variação: 1 a $8,2 \mathrm{~kg}$ ), com redução da gordura truncal de $36,8 \pm 10,1 \%$ para $33,1 \pm 9,4 \%(\mathrm{p}<0,01)$, correspondente à redução média de $1,9 \mathrm{~kg}$ (variação $0,6 \mathrm{a}$ $6,9 \mathrm{~kg}$ ). Em associação, houve aumento significativo da massa magra, cujos valores basais eram de 43,7 $\pm 13,0 \mathrm{~kg}$, atingindo o pico ao final do $2^{\circ}$ ano de tratamento com aumento médio de $2,6 \mathrm{~kg}$, e chegando ao final do estudo com ganho médio de $0,8 \mathrm{~kg}(\mathrm{p}<0,01)$ (Tabela $\mathrm{l})$.

\section{Efeitos do tratamento com GH na densidade mineral óssea}

A análise foi realizada em 14 casos, pois quatro pacientes não realizaram avaliação da DMO no final do estudo. Houve aumento da DMO na coluna lombar $\left(1,064 \pm 0,2\right.$ versus $\left.1,101 \pm 0,2 \mathrm{~g} / \mathrm{cm}^{2} ; \mathrm{p}=0,03\right) \mathrm{e}$ no fêmur total $\left(0,961 \pm 0,2\right.$ versus $1,00 \pm 0,2 \mathrm{~g} / \mathrm{cm}^{2}$; $\mathrm{p}=0,01)$, com valores no colo de fêmur muito próximos à significância estatística $(0,908 \pm 0,16$ versus $\left.0,938 \pm 0,15 \mathrm{~g} / \mathrm{cm}^{2} ; \mathrm{p}=0,056\right)$. No início do estudo, o T-escore da DMO estava alterado (<-1 DP) em pelo menos um dos sítios em nove $(50 \%)$ pacientes. Nestes casos, o T-escore médio melhorou significativamente, passando de $-2,06 \pm 0,62$ para $-1,66 \pm 0,78$ $(\mathrm{p}=0,04)($ Figura $\mathrm{l})$.

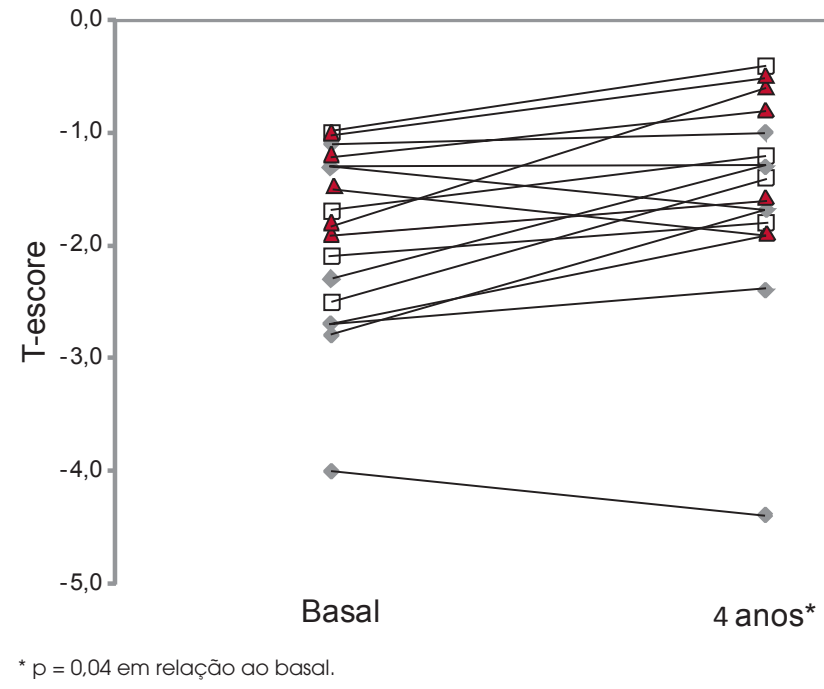

Figura 1. Efeitos de quatro anos de tratamento de reposição com GH sobre a densidade mineral óssea nos sítios ósseos com T-escore menor que 1,0 na avaliação inicial em pacientes com DGHA. Coluna lombar representada por losangos cinza, colo do fêmur representado por triângulos vermelho e fêmur total representado por quadrados abertos.

\section{Efeitos do tratamento com GH nos parâmetros laboratoriais}

A Tabela 2 resume as variações observadas nos principais parâmetros laboratoriais. Ao final do primeiro ano de tratamento com dose fixa de $\mathrm{GH}$, ocorreu aumento dos níveis de insulina e piora do HOMA-IR, mas sem mudanças significativas das glicemias de jejum. Ao final do quarto ano de tratamento, os níveis de insulina não diferiram dos valores pré-tratamento $(10,8 \pm 4,9 \mu \mathrm{UI} / \mathrm{mL}$ versus $8,7 \pm 8,9 \mu \mathrm{UI} / \mathrm{mL}$ ), e as variações na insulinemia se correlacionaram com as variações na gordura total $(\mathrm{r}=$ $0,52 ; \mathrm{p}=0,03)$. Similarmente, os valores do HOMA-IR ao final do tratamento não eram diferentes dos basais $(2,3 \pm 1,4$ versus $1,9 \pm 2,2)$. Não ocorreram variações significativas nas glicemias e na hemoglobina glicosilada. Dois pacientes que apresentavam glicemias de jejum alteradas no início do tratamento (valores de 103 e 114 $\mathrm{mg} / \mathrm{dL}$ ) desenvolveram diabetes tipo 2 no seguimento. Houve redução significativa do colesterol total $(222,0 \pm$ $45,5 \mathrm{mg} / \mathrm{dL}$ versus $197,1 \pm 38,0 \mathrm{mg} / \mathrm{dL} ; \mathrm{p}<0,01)$ e do LDL-colesterol $(147,5 \pm 49,5 \mathrm{mg} / \mathrm{dL}$ versus $115,1 \pm$ $28,6 \mathrm{mg} / \mathrm{dL} ; \mathrm{p}<0,01)$, mas não houve mudanças nos níveis de triglicerídeos e HDL-colesterol. Quanto mais altos os valores do colesterol total e do LDL-colesterol e mais baixos os do HDL-colesterol antes do tratamento, maiores as variações observadas nestes parâmetros ao final do estudo $(\mathrm{r}=0,75 ; \mathrm{p}<0,01)$. 
Tabela 2. Efeitos de quatro anos de tratamento de reposição com GH sobre os parâmetros laboratoriais em 18 pacientes com DGHA.

\begin{tabular}{|c|c|c|c|c|c|}
\hline & Basal & $1^{\circ}$ ano & $2^{\circ}$ ano & $3^{\circ}$ ano & $4^{\circ}$ ano \\
\hline Glicose (mg/dL) & $84,6 \pm 12,6$ & $97,5 \pm 41,1$ & $89,3 \pm 14,4$ & $87,7 \pm 10,0$ & $87,8 \pm 11,3$ \\
\hline Insulina $(\mu \mathrm{UI} / \mathrm{mL})$ & $10,8 \pm 4,9$ & $17,0 \pm 14,0^{a}$ & $14,8 \pm 15,5$ & $9,7 \pm 7,8$ & $8,7 \pm 8,9$ \\
\hline HOMA-IR & $2,3 \pm 1,4$ & $4,4 \pm 4,6^{a}$ & $3,5 \pm 4,7$ & $2,1 \pm 1,9$ & $1,9 \pm 2,2$ \\
\hline Colesterol (mg/dL) & $222,0 \pm 45,5$ & $209,6 \pm 52,4$ & $212,5 \pm 72,1$ & $198,5 \pm 35,2^{b}$ & $197,1 \pm 38,0^{\circ}$ \\
\hline $\mathrm{HDL}(\mathrm{mg} / \mathrm{dL})$ & $48,0 \pm 11,7$ & $46,4 \pm 10,7$ & $48,4 \pm 11,9$ & $46,0 \pm 9,5$ & $51,5 \pm 8,5$ \\
\hline $\mathrm{LDL}(\mathrm{mg} / \mathrm{dL})$ & $147,5 \pm 49,5$ & $123,3 \pm 40,9 c$ & $134,8 \pm 67,7$ & $114,6 \pm 32,1^{b}$ & $115,1 \pm 28,6^{b}$ \\
\hline Triglicerídeos (mg/dL) & $148,4 \pm 80,4$ & $156,1 \pm 111,4$ & $163,9 \pm 77,5$ & $178,4 \pm 101,6$ & $154,4 \pm 93,2$ \\
\hline
\end{tabular}

${ }^{a} \mathrm{p}=0,01$ versus basal; ${ }^{\mathrm{b}} \mathrm{p} \leq 0,001$ versus basal; ${ }^{\mathrm{c}} \mathrm{p}=0,03$ versus basal

\section{Efeitos do tratamento com GH na estrutura e função cardíacas}

Nenhuma anormalidade significativa foi encontrada na avaliação pré-tratamento e não foram observadas mudanças significativas após quatro anos de terapia com GH (Tabela 3).

\section{Correlações entre as mudanças nos níveis de IGF-1, composição corporal e parâmetros metabólicos}

Não foram encontradas correlações entre as mudanças nos níveis absolutos de IGF-1 e IGF-1 DP com as alterações na gordura corporal, massa magra, DMO e parâmetros bioquímicos.

Tabela 3. Efeitos de quatro anos de tratamento de reposição com $\mathrm{GH}$ sobre parâmetros ecocardiográficos em 18 pacientes com DGHA.

\begin{tabular}{|c|c|c|}
\hline & Basal & 4 anos \\
\hline Dimensão da raiz aórtica (mm) & $29,2 \pm 3,8$ & $30,2 \pm 3,8$ \\
\hline Dimensão do AE (mm) & $34,5 \pm 4,9$ & $35,4 \pm 5,5$ \\
\hline Dimensão diastólica do VD (mm) & $17,5 \pm 3,3$ & $16,3 \pm 4,4$ \\
\hline Espessura diastólica do SV (mm) & $10,0 \pm 2,4$ & $9,8 \pm 1,4$ \\
\hline $\begin{array}{l}\text { Dimensão diastólica da parede } \\
\text { posterior do VE (mm) }\end{array}$ & $10,0 \pm 2,3$ & $9,5 \pm 1,2$ \\
\hline Dimensão diastólica do VE (mm) & $43,4 \pm 7,0$ & $46,0 \pm 6,4$ \\
\hline Dimensão sistólica do VE (mm) & $25,0 \pm 5,6$ & $29,0 \pm 4,0$ \\
\hline $\begin{array}{l}\text { Encurtamento percentual } \\
\text { do VE (\%) }\end{array}$ & $43,5 \pm 6,6$ & $37,2 \pm 3,5$ \\
\hline Fração de ejeção (\%) & $71,3 \pm 8,2$ & $66,8 \pm 4,4$ \\
\hline Massa do VE (g) & $151,0 \pm 58,2$ & $162,0 \pm 53,0$ \\
\hline Índice de massa do VE ( $\left.\mathrm{g} / \mathrm{m}^{2}\right)$ & $86,6 \pm 24,0$ & $110,5 \pm 55,4$ \\
\hline
\end{tabular}

$\mathrm{AE}$ = átrio esquerdo; $\mathrm{VD}$ = ventrículo direito; $\mathrm{SV}$ = septo ventricular; $\mathrm{VE}=$ ventrículo esquerdo.

\section{Efeitos colaterais e eventos adversos}

Relataram efeitos colaterais três pacientes. No início do tratamento, a paciente portadora de mutação no Pit-1 apresentou edema e artralgia, e outra paciente com hipopituitarismo congênito apresentou edema. Houve redução da dose no primeiro caso, com aumento posterior de modo mais progressivo e lento sem que ocorresse o reaparecimento dos eventos adversos, enquanto no segundo caso as queixas desapareceram após duas semanas sem ajuste na dosagem do GH. Outro paciente apresentou cefaléia após aumento da dose de 0,4 para $0,5 \mathrm{mg} /$ dia, necessitando de reajuste da dose temporariamente, mas sem ter interrompido o tratamento. Não houve nenhum caso de recorrência ou crescimento de lesão residual nos sete pacientes com história de tumores hipotalâmico-hipofisários.

\section{DISCUSSÃo}

Os efeitos do GH sobre a composição corporal de pacientes com DGHA podem variar de acordo com a duração do tratamento. Uma análise dos estudos cujo período de tratamento foi de dois a 36 meses mostrou redução média de 4 a $6 \mathrm{~kg}$ da gordura corporal total e aumento médio de 2 a $5,5 \mathrm{~kg}$ na massa magra (2), porém estes achados não são consistentes nos estudos com duração igual ou superior a quatro anos. Arwert e cols. (21) observaram redução da relação cintura-quadril após quatro anos de tratamento com $\mathrm{GH}$, porém esta relação aumentou significativamente junto com o aumento do IMC após dez anos de acompanhamento. Gibney e cols. (22) avaliaram os efeitos de dez anos da reposição de GH na DGHA e encontraram aumento de $4,9 \mathrm{~kg}$ na massa magra, mas não observaram mudanças significativas na gordura corporal. Em uma avaliação de 
cinco anos, Gotherstrom e cols. (23) demonstraram redução de $1,2 \mathrm{~kg}$ na gordura corporal e aumento de 2,3 kg na massa magra após cinco anos tratamento, mas estes efeitos foram neutralizados pela maior idade e ganho de peso dos pacientes quando a avaliação foi repetida após dez anos de seguimento (24). Nossos resultados foram intermediários entre os achados destes estudos, com redução média de $2,8 \mathrm{~kg}$ na gordura corporal total e $1,9 \mathrm{~kg}$ na gordura truncal, ganho de $0,8 \mathrm{~kg}$ na massa magra e nenhuma variação no peso, IMC e medidas antropométricas. Vários fatores poderiam explicar estas diferenças, incluindo a história natural de ganho de peso com a idade, população estudada, aderência ao tratamento, dose e variação individual na resposta terapêutica.

A dose e o regime de tratamento com $\mathrm{GH}$ variam bastante entre os estudos, com implicações sobre os níveis séricos de IGF-1 que são mantidos ao longo do tratamento. A dose média utilizada pelos nossos pacientes foi de $0,45 \mathrm{mg} /$ dia, similar à dose média de $0,48 \mathrm{mg} /$ dia relatadas nos estudos de Gotherstrom e cols. $(23,24)$ e de $0,4 \mathrm{mg} /$ dia usada por Arwert e cols. (21), mas menor que a dose de $0,61 \mathrm{mg} /$ dia utilizada no estudo de Gibney e cols. (22). Apesar de várias limitações, as dosagens séricas de IGF-1 permanecem como a melhor forma de adequação do tratamento com GH, procurando-se manter os níveis entre a mediana e o limite superior do normal dos valores de referência. Entretanto, as variações nos níveis de IGF-l não se relacionam com os efeitos do tratamento com GH sobre a composição corporal e fatores de risco cardiovasculares (1-4,25-27). Nos nossos pacientes, tanto no primeiro ano de reposição com dose baixa e fixa, em que não se obteve normalização dos níveis de IGF-1 em uma grande proporção de pacientes (26), quanto após três anos de tratamento com dose titulada, os efeitos do GH sobre a composição corporal, massa óssea e perfil lipídico não foram relacionados com as variações nos níveis de IGF-1. Assim, as dosagens de IGF-1 são mais adequadas para evitar dose excessiva de GH durante o tratamento do que para julgar os efeitos desta terapia $(1-4,25,27)$. Além disso, os níveis de IGF- 1 podem ser úteis para avaliar a aderência ao tratamento. Em aproximadamente um terço dos nossos pacientes, os níveis séricos de IGF-1 estavam baixos ao final do quarto ano, possivelmente refletindo má aderência ao tratamento, fator este que deve ser sempre considerado em qualquer estudo que objetive avaliar eficácia e segurança de terapias prolongadas.
O GH desempenha papel importante na aquisição de massa óssea e influencia o processo de remodelação óssea. Os efeitos da DGHA sobre a massa óssea são dependentes da idade, com maior comprometimento nos pacientes mais jovens e com DGHA instalada na infância $(2-4,13,28)$. No presente estudo, a média de idade foi de 50,5 anos e a DGHA iniciou-se na vida adulta em quase $80 \%$ do grupo. Por isso, somente metade dos nossos pacientes apresentava comprometimento da DMO em algum sítio ósseo na fase pré-tratamento. Observamos aumento significativo da DMO tanto na coluna lombar quanto no fêmur, mas mais importante, houve melhora nos sítios estudados cujo T-escore era menor do que $-1,0$ na avaliação inicial. Nossos achados estão de acordo com os trabalhos da literatura que demonstram que a reposição de GH por tempo prolongado pode melhorar ou normalizar a DMO na coluna lombar e no fêmur (29-32), particularmente nos pacientes que apresentam comprometimento antes do início da reposição com GH. Nas mulheres, a reposição adequada de estrogênio parece ser fundamental para que sejam detectados os efeitos benéficos do GH sobre a massa óssea (29).

O envelhecimento se associa com redução na sensibilidade dos tecidos à ação da insulina e sugere-se que a terapia com GH na DGHA poderia prevenir a deterioração da sensibilidade insulínica associada com a idade, por promover redução da gordura corporal (10). Entretanto, o GH também tem efeitos diabetogênicos e estas ações antagônicas dificultam a interpretação dos potenciais efeitos que a terapia prolongada possa ter sobre o metabolismo intermediário. Uma metanálise de estudos controlados com placebo com duração de até 18 meses demonstrou que a terapia com GH se associa com elevações nos níveis de glicose e insulina de jejum e redução da sensibilidade insulínica (33). Em dois estudos em que o tratamento se prolongou por cinco anos foram observados aumento da glicemia de jejum, sem alteração ou com leve elevação nos níveis de insulina, manutenção do HOMA-IR e redução da hemoglobina glicosilada $(7,23)$. Em um estudo brasileiro com o mesmo tempo de observação, Cenci e cols. (34) não observaram mudanças nos níveis de glicose, insulina e no índice HOMA e relataram elevação inicial das glicemias médias coletadas duas horas após sobrecarga oral de glicose, seguida de redução nos dois últimos anos de tratamento. No estudo inglês com dez anos de seguimento, não houve alteração significativa nos níveis de glicose ou insulina (22), ao passo que no estudo 
sueco os níveis glicêmicos se elevaram, os de insulina não se alteraram e a hemoglobina glicosilada reduziu após dez anos de tratamento (24). Nossos resultados foram bastante favoráveis e similares aos da literatura, com piora dos níveis de insulina e do índice HOMA-IR ao final do primeiro ano de tratamento, mas com normalização após este período, visto que, ao final do período de observação, os valores de glicose, insulina, HOMA-IR e hemoglobina glicosilada não diferiram daqueles encontrados no pré-tratamento. Entretanto, dois pacientes que apresentavam glicemia de jejum alterada desenvolveram diabetes no primeiro ano do estudo, mostrando que em casos individuais pode haver agravamento da tolerância à glicose.

Um fator que parece ser de grande importância para os efeitos finais na sensibilidade insulínica é a dose de GH utilizada ao longo do tratamento. No estudo de Arwert e cols. (2l), a dose média variou entre $0,97 \mathrm{mg} /$ dia a $0,71 \mathrm{mg} /$ dia ao longo de quatro anos de tratamento, e esteve associada com o aumento da glicemia e da hemoglobina glicosilada. Igualmente, 8 de 17 pacientes estudados por Spina e cols. (35) desenvolveram intolerância à glicose após dois anos de tratamento com doses médias de 0,72 e $0,84 \mathrm{mg} /$ dia de GH. Nestes dois estudos, a dose média de $\mathrm{GH}$ foi pelo menos $60 \%$ mais alta do que aquela administrada aos nossos pacientes e em outros estudos de longo prazo. Em conjunto, as informações disponíveis enfatizam a importância da monitoração contínua dos pacientes com DGHA para o desenvolvimento de intolerância à glicose e diabetes tipo 2 , especialmente naqueles com alterações glicêmicas prétratamento e nos que recebem doses médias elevadas de $\mathrm{GH}$ durante terapia prolongada.

Um achado mais consistente, tanto nos estudos de curta quanto de longa duração, é a mudança do perfil lipídico resultante do tratamento com $\mathrm{GH}$, especialmente a redução do colesterol total e do LDL-colesterol (1-4,21-24). Na nossa casuística, apenas três pacientes apresentavam perfil lipídico normal na avaliação basal e, em consonância com outros trabalhos da literatura, houve melhora significativa do colesterol total e LDL-colesterol. Os pacientes com colesterol total e LDL mais altos e HDL mais baixos no início apresentaram mudanças mais expressivas nestes parâmetros, mostrando que quanto mais desfavorável o perfil lipídico pré-tratamento mais pronunciada é a resposta à reposição com $\mathrm{GH}$. Essas mudanças no perfil lipídico não podem ser atribuídas ao uso de medicações hipolipemiantes associadas, visto que as intervenções nesse sentido foram mínimas.
Vários índices de estrutura e função cardíacas podem estar alterados na DGHA, embora a maioria dos pacientes não apresente sintomas $(15,36,37)$. As anormalidades são mais proeminentes na DGHA de instalação na infância do que na vida adulta e se caracterizam por redução do tamanho do coração, estreitamento de paredes cardíacas, redução do enchimento diastólico e comprometimento da função sistólica (37). Entretanto, esses achados não são consistentes em todos os estudos e muitas vezes dependem do momento em que a avaliação é realizada. Em um estudo com 38 homens com DGHA de início na infância, ter Maaten e cols. encontraram aumento significativo da massa cardíaca no primeiro ano do estudo, mas este aumento não se manteve após dois anos de tratamento, porquanto, ao final do estudo, a massa cardíaca era semelhante àquela do pré-tratamento (38). Muitos estudos não-controlados e de curta duração demonstram melhora da função sistólica após tratamento com GH em crianças e adultos com DGH $(37,39,40)$, porém este efeito tem sido questionado por estudos controlados com placebo (41). Há poucos dados sobre os efeitos da reposição de longa duração com GH sobre o coração. No nosso grupo de pacientes não observamos variações significativas em nenhum dos parâmetros ecocardiográficos avaliados após quatro anos de tratamento, em acordo com dois outros estudos com sete e dez anos de acompanhamento $(22,42)$. O fato de o nosso grupo consistir basicamente de pacientes com DGHA iniciada na vida adulta deve ter contribuído para a ausência de alterações ecocardiográficas.

Em resumo, este estudo prospectivo, longitudinal, aberto e não-controlado leva à conclusão de que quatro anos de tratamento de reposição com dose média de $0,44 \mathrm{mg} /$ dia de $\mathrm{GH}$ em pacientes com DGHA promove mudanças positivas na composição corporal, caracterizadas por redução da gordura corporal total e gordura truncal, aumento da massa magra e melhora da DMO na coluna lombar e no fềmur, esta última de particular importância nos pacientes com DMO baixa antes do início da terapia. Este tratamento teve impacto positivo sobre o perfil lipídico e não afetou a sensibilidade insulínica e a função cardíaca. Entretanto, monitoração cuidadosa da glicemia é fundamental, especialmente em pacientes com intolerância à glicose pré-tratamento e naqueles que recebem doses médias elevadas de GH por tempo prolongado. A reposição de GH é segura, com poucos eventos adversos, mas a aderência ao tratamento prolongado com injeções subcutâneas diárias é um problema que deve ser constantemente monitorado na prática clínica. 


\section{AGRADECIMENTOS}

À Novo Nordisk Farmacêutica do Brasil (São Paulo, Brasil) pelo fornecimento gratuito do GH recombinante humano (Norditropin e Norditropin simplexx ${ }^{\circledR}$ ) durante todo o período do estudo. Ao Conselho Nacional de Desenvolvimento Científico e Tecnológico (CNPq) pelo apoio ao projeto por meio de concessão de bolsa de produtividade em pesquisa ao Dr. Cesar Luiz Boguszewski. Ao Dr. Victor Fernando Lopez Arce e às bioquímicas Gislaine Custodio Piovezan e Flavia Shibata pelo suporte técnico ao longo do estudo.

\section{REFERÊNCIAS}

1. Hartman ML. The Growth Hormone Research Society consensus guidelines for the diagnosis and treatment of adult $\mathrm{GH}$ deficiency. Growth Horm IGF Res. 1998;8 Suppl A:25-9.

2. Simpson H, Savine R, Sonksen $P$, Bengtsson BA, Carlsson L, Christiansen JS, et al. GRS council. Growth hormone replacement therapy for adults: into the new millennium. Growth Horm. IGF Res. 2002;12:1-33.

3. Molitch ME, Clemmons DR, Malozowski S, Merriam GR, Shalet SM, Vance ML. Endocrine Society's Clinical Guidelines Subcommittee, Stephens PA. Evaluation and treatment of adult growth hormone deficiency: an endocrine society clinical practice guideline. $\mathrm{J}$ Clin Endocrinol Metab. 2006;91:1621-34.

4. Ho KK, 2007 GH Deficiency Consensus Workshop Participants. Consensus guidelines for the diagnosis and treatment of adults with $\mathrm{GH}$ deficiency II: a statement of the $\mathrm{GH}$ research society in association with the european society for pediatric endocrinology, lawson Wilkins Society, European Society of Endocrinology, Japan Endocrine Society, and Endocrine Society of Australia. Eur J Endocrinol. 2007;157:695-700.

5. Yuen KC, Dunger DB. Therapeutic aspects of growth hormone and insulin-like growth factor-I treatment on visceral fat and insulin sensitivity in adults. Diabetes Obes Metab. 2007;9(1):11-22.

6. Svensson J; Fowelin J; Landin K, Bengtsson BA, Johansson JO. Effect of seven years of GH-replacement therapy on insulin sensitivity in GH-deficient adults. J Clin Endocrinol Metab. 2002;87:2121-7.

7. Giavoli C, Porretti S, Ronchi CL, Cappiello V, Ferrante E, Orsi E, et al. Long-term monitoring of insulin sensitivity in growth hormone-deficient adults on substitutive recombinant human growth hormone therapy. Metabolism. 2004;53:740-3.

8. Christopher M, Hew FL, Oakley M, Rantzau C, Alford F. Defects of insulin action and skeletal muscle glucose metabolism in growth hormone-deficient adults persist after 24 months of recombinant human growth hormone therapy. J Clin Endocrinol Metab. 1998;83:1668-81.

9. Jørgensen JO, Nørrelund H, Conceicao F, Møller N, Christiansen JS. Somatropin and glucose homeostasis: considerations for patient management. Treat Endocrinol. 2002;1:229-334.

10. Svensson J, Bengtsson BA. Growth hormone replacement therapy and insulin sensitivity. J Clin Endocrinol Metab. 2003;88(4):1453-4.
11. Murray RD, Columb B, Adams JE, Shalet SM. Low bone mass is an infrequent feature of the adult Growth hormone deficiency syndrome on middle-age adults and the elderly. J Clin Endocrinol Metab. 2004;89:1124-30.

12. Wüster $C$, Abs R, Bengtsson BA, Bennmarker H, Feldt-Rasmussen $U$, Hernberg-Ståhl $E$, et al. The influence of growth hormone deficiency, growth hormone replacement therapy, and other aspects of hypopituitarism on fracture rate and bone mineral density. J Bone Miner Res. 2001;16(2):398-405.

13. Ohlsson C, Bengtsson BA, Isaksson OG, Andreassen TT, Slootweg MC. Growth hormone and bone. Endocr Rev. 1998;19(1):55-79.

14. Rosén T, Bengtsson BA. Premature mortality due to cardiovascular disease in hypopituitarism. Lancet. 1990;336:285-8.

15. Burger AG, Monson JP, Colao AM, Klibanski A. Cardiovascular risk in patients with growth hormone deficiency: effects of growth hormone substitution. Endocr Pract. 2006;12:682-9.

16. Gillberg P, Bramnert M, Thorén M, Werner S, Johannsson G. Commencing growth hormone replacement in adults with a fixed low dose: effects on serum lipoproteins, glucose metabolism, body composition, and cardiovascular function. Growth Horm IGF Res. 2001;11:273-81.

17. Friedewald WT, Levy RI, Fredrickson DS. Estimation of the concentration of low-density lipoprotein cholesterol in plas$\mathrm{ma}$, without use of the preparative ultracentrifuge. Clin Chem. 1972;18:499-502.

18. Matthews DR, Hosker JP, Rudenski AS, Naylor BA, Treacher DF, Turner RC. Homeostasis model assessment: insulin resistance and beta-cell function from fasting plasma glucose and insulin concentrations in man. Diabetologia. 1985;28:412-9.

19. Sahn DJ, DeMaria A, Kisslo J, Weyman A. Recommendations regarding quantitation in M-mode echocardiography: results of a survey of echocardiographic measurements. Circulation. 1978;58:1072-83.

20. Devereux RB, Reichek N. Echocardiographic determination of left ventricular mass in man. Anatomic validation of the method. Circulation. 1977;55:613-8.

21. Arwert LI, Ross JC, Lips P, Twisk JW, Manoliu RA, Drent ML. Effects of 10 years of growth hormone $(\mathrm{GH})$ replacement therapy in adult GH-deficient men. Clin Endocrinol (Oxf). 2005;63:310-6.

22. Gibney J, Wallace JD, Spinks T, Schnorr L, Ranicar A, Cuneo $\mathrm{RC}$, et al. The effects of 10 years of recombinant human growth hormone (GH) in adult GH-deficient patients. J Clin Endocrinol Metab. 1999;84:2596-2602.

23. Gotherstrom G, Svensson J, Koranyi J, Alpsten M, Bosaeus I, Bengtsson B, et al. A prospective study of 5 years of $\mathrm{GH}$ replacement therapy in $\mathrm{GH}$-deficient adults: sustained effects on body composition, bone mass, and metabolic indices. J Clin Endocrinol Metab. 2001;86:4657-65.

24. Gotherstrom G, Bengtsson BA, Bosaeus I, Johannsson G, Svensson J. A 10-year, prospective study of the metabolic effects of growth hormone replacement in adults. J Clin Endocrinol Metab. 2007;92(4):1442-5.

25. Clemmons DR. Clinical utility of measurements of insulin-like growth factor 1. Nat Clin Pract Endocrinol Metab. 2006;2:436-46.

26. Boguszewski CL, Meister LH, Zaninelli DC, Radominski RB. One year of GH replacement therapy with a fixed low-dose regimen improves body composition, bone mineral density and lipid profile of $\mathrm{GH}$-deficient adults. Eur $\mathrm{J}$ Endocrinol. 2005;152:67-75.

27. Monson JP. Monitoring of insulin-like growth factors during growth hormone treatment: adulthood growth hormone deficiency. Endocr Rev. 2005;9:89-99. 
28. Murray RD, Columb B, Adams JE, Shalet SM. Low bone mass is an infrequent feature of the adult growth hormone deficiency syndrome in middle-age adults and the elderly. J Clin Endocrinol Metab. 2004;89:1124-30.

29. Götherström G, Bengtsson BA, Bosaeus I, Johannsson G, Svensson J. Ten-year GH replacement increases bone mineral density in hypopituitary patients with adult onset GH deficiency. Eur J Endocrinol. 2007;156:55-64.

30. Biermasz NR, Hamdy NA, Pereira AM, Romijn JA, Roelfsema F. Long-term skeletal effects of recombinant human growth hormone (hrGH) alone and $\mathrm{hrGH}$ combined with alendronate in GH-deficient adults: a seven-year follow-up study. Clin Endocrinol (Oxf). 2004;60:568-75.

31. Bravenboer N, Holzmann PJ, ter Maaten JC, Stuurman LM, Roos JC, Lips P. Effect of long-term growth hormone treatment on bone mass and bone metabolism in growth hormone-deficient men. J Bone Miner Res. 2005;20:1778-84.

32. Wilhelm B, Kann PH. Long-term effects of 7-year growth hormone substitution on bone metabolism, bone density, and bone quality in growth hormone-deficient adults. Med Klin (Munich). 2004;99:569-77.

33. Maison P, Griffin S, Nicoue-Beglah M, Haddad N, Balkau B, Chanson P. Impact of growth hormone (GH) treatment on cardiovascular risk factors in $\mathrm{GH}$-deficient adults: a meta-analysis of blinded, randomized, placebo-controlled trials. J Clin Endocrinol Metab. 2004;89:2192-9.

34. Cenci MC, Conceição FL, Soares DV, Spina LD, Brasil RR, Lobo PM, et al. Impact of 5 years of growth hormone replacement therapy on cardiovascular risk factors in growth hormone-deficient adults. Metabolism. 2008;57:121-9.

35. Spina LD, Soares DV, Brasil RR, Lobo PM, Conceição F, Vaisman M. Glucose metabolism and visceral fat in $\mathrm{GH}$ deficient adults: two years of GH-replacement. Pituitary. 2004;7:123-9.

36. Beshyah SA, Johnston DG. Cardiovascular disease and risk factors in adults with hypopituitarism. Clin Endocrinol (Oxf). 1999:50:1-15.
37. Colao A, Marzullo P, Di Somma C, Lombardi G. Growth hormone and the heart. Clin Endocrinol (Oxf). 2001;54:137-54.

38. ter Maaten JC, de Boer H, Kamp O, Stuurman L, van der Veen EA. Long-term effects of growth hormone $(\mathrm{GH})$ replacement in men with childhood-onset GH deficiency. J Clin Endocrinol Metab. 1999;84:2373-80.

39. Cuocolo A, Nicolai E, Colao A, Longobardi S, Cardei S, Fazio S, et al. Improved left ventricular function after growth hormone replacement in patients with hypopituitarism: assessment with radionuclide angiography. Eur J Nucl Med. 1996;23:390-4.

40. Shulman DI, Root AW, Diamond FB, Bercu BB, Martinez R, Boucek RJ Jr. Effects of one year of recombinant human growth hormone $(\mathrm{GH})$ therapy on cardiac mass and function in children with classical GH deficiency. J Clin Endocrinol Metab. 2003;88:4095-9.

41. Sneppen SB, Steensgaard-Hansen F, Feldt-Rasmussen U. Cardiac effects of low-dose growth hormone replacement therapy in growth hormone-deficient adults: an 18-month randomised, placebo-controlled, double-blind study. Horm Res. 2002;58(1):21-9.

42. Chrisoulidou A, Beshyah SA, Rutherford O, Spinks TJ, Mayet $\mathrm{J}$, Kyd P, et al. Effects of 7 years of growth hormone replacement therapy in hypopituitary adults. J Clin Endocrinol Metab. 2000;85:3762-9.

\section{Endereço para correspondência:}

Cesar Luiz Boguszewski

SEMPR do Hospital de Clínicas - UFPR

Av. Agostinho Leão Junior, 262

80030-110 Curitiba, PR

E-mail: cesarluiz@hc.ufpr.br 